International Journal of Pure and Applied Mathematics Volume 109 No. 1 2016, 85-104

ISSN: 1311-8080 (printed version); ISSN: 1314-3395 (on-line version)

url: http://www.ijpam.eu

doi: 10.12732 /ijpam.v109i1.7

ijpam.eu

\title{
MULTIPLE PERIODIC SOLUTIONS FOR PERTURBED SECOND-ORDER IMPULSIVE HAMILTONIAN SYSTEMS
}

\author{
John R. Graef ${ }^{1}$, Shapour Heidarkhani ${ }^{2}$, Lingju Kong ${ }^{1}$ \\ ${ }^{1}$ Department of Mathematics \\ University of Tennessee at Chattanooga \\ Chattanooga, TN 37403, USA \\ e-mail: John-Graef@utc.edu \\ ${ }^{2}$ Department of Mathematics \\ Faculty of Sciences \\ Razi University \\ Kermanshah 67149, IRAN
}

\begin{abstract}
The existence of three distinct periodic solutions for a class of perturbed impulsive Hamiltonian systems is established. The techniques used in the proofs are based on variational methods.
\end{abstract}

AMS Subject Classification: $34 \mathrm{~B} 15$

Key Words: multiple periodic solutions, perturbed Hamiltonian system, impulsive systems, critical point theory, variational methods

Received: August 1, 2016

Published: September 1, 2016
(C) 2016 Academic Publications, Ltd. url: www.acadpubl.eu 


\section{Introduction}

We consider the perturbed Hamiltonian system

$$
\left\{\begin{aligned}
-\ddot{u}(t)+A(t) u(t)=\lambda \nabla F(t, & u(t))+\mu \nabla G(t, u(t)) \\
& +\nabla H(u(t)), \text { a.e. } t \in[0, T], \\
\Delta\left(\dot{u}_{i}\left(t_{j}\right)\right)=I_{i j}\left(u_{i}\left(t_{j}\right)\right), \quad i= & 1,2, \ldots, N, j=1,2, \ldots, p, \\
u(0)-u(T)=\dot{u}(0)-\dot{u}(T)= & 0
\end{aligned}\right.
$$

where $T>0, N \geq 1, p \geq 2, \lambda>0$ and $\mu \geq 0$ are parameters, $A:[0, T] \rightarrow \mathbb{R}^{N \times N}$ is a continuous map from the interval $[0, T]$ to the set of $N \times N$ symmetric matrices, $t_{j}, j=1,2, \ldots, p$, are the instants at which the impulses occur, $0=t_{0}<t_{1}<\ldots<t_{p}<t_{p+1}=T$, and $\Delta\left(\dot{u}_{i}\left(t_{j}\right)\right)=\dot{u}_{i}\left(t_{j}^{+}\right)-\dot{u}_{i}\left(t_{j}^{-}\right)=$ $\lim _{t \rightarrow t_{j}^{+}} \dot{u}_{i}(t)-\lim _{t \rightarrow t_{j}^{-}} \dot{u}_{i}(t)$. Here, $I_{i j}: \mathbb{R} \rightarrow \mathbb{R}$ are Lipschitz continuous with Lipschitz constants $L_{i j}>0$, i.e.,

$$
\left|I_{i j}\left(s_{1}\right)-I_{i j}\left(s_{2}\right)\right| \leq L_{i j}\left|s_{1}-s_{2}\right|
$$

for every $s_{1}, s_{2} \in \mathbb{R}$, and $I_{i j}(0)=0$ for $i=1,2, \ldots, N, j=1,2, \ldots, p$. In addition, $F, G:[0, T] \times \mathbb{R}^{N} \rightarrow \mathbb{R}$ are measurable with respect to $t$ for all $u \in \mathbb{R}^{N}$, continuously differentiable in $u$ for almost every $t \in[0, T]$, and satisfy the standard summability condition

$$
\sup _{|x| \leq a}(\max \{|F(\cdot, x)|,|\nabla F(\cdot, x)|,|G(\cdot, x)|,|\nabla G(\cdot, x)|\}) \in L^{1}([0, T])
$$

for any $a>0$. The function $H: \mathbb{R}^{N} \rightarrow \mathbb{R}$ is continuously differentiable, $H(0, \ldots, 0)=0, \nabla H$ is Lipschitz continuous with Lipschitz constant $L>0$, i.e.,

$$
\left|\nabla H\left(x_{1}\right)-\nabla H\left(x_{2}\right)\right| \leq L\left|x_{1}-x_{2}\right|
$$

for every $x_{1}, x_{2} \in \mathbb{R}^{N}$, and $\nabla H(0, \ldots, 0)=0$. If we assume that $\nabla F, \nabla G$ : $[0, T] \times \mathbb{R}^{N} \rightarrow \mathbb{R}$ are continuous, then condition (2) is satisfied.

As is well known, a Hamiltonian system is a system of differential equations that can model the motion of a mechanical system. An important and interesting question is under what conditions will the Hamiltonian system possess 
periodic solutions. Background information and applications of Hamiltonian systems can be found for example in [16, 28, 31, 37]. The monographs [29, 32] have inspired a great deal of work on the existence and multiplicity of periodic solutions for Hamiltonian systems using variational techniques; for example, see $[9,10,11,13,14,15,18,19,24,25,26,36,38,40,42,43,45]$ and the references therein.

Impulsive differential equations provide a general framework for modeling many real world phenomena; they too have been studied extensively in the literature. Background information and applications of impulsive differential equations can be found in $[2,3,23,27,33]$. Recently, using critical point theory, several authors have studied the existence and multiplicity of solutions of impulsive problems; see, for example, [1, 7, 20, 30, 39, 41].

The existence and multiplicity of solutions for second-order impulsive Hamiltonian systems have attracted a good deal of attention in the literature, and we refer the reader to $[12,34,35,44]$ and the included references for recent results. In $[12,35]$, using variational methods and critical point theory, the existence of multiple solutions for second-order impulsive Hamiltonian systems was studied. In [21], using different variational techniques from the ones used in this paper, the present authors obtained the existence of infinitely many classical periodic solutions to problem (1); in [22], using variational methods and critical point theory different from those in [21] and this paper, they investigated the existence of nontrivial periodic solutions to problem (1) in case $\mu=0$.

Motivated by the results in $[12,35]$ and using two kinds of three critical points theorems (Theorems 1 and 2 below), in this paper we are able ensure the existence of at least three classical periodic solutions to problem (1); see Theorems 5 and 6 below. Theorems 1 and 2 have been successfully employed to establish the existence of at least three solutions for perturbed boundary value problems in the papers $[5,6,17]$.

\section{Preliminaries}

Our main tools are the three critical point theorems that we recall here in convenient forms. The first has been obtained in [4], and it is a more precise version of Theorem 3.2 of [8]. The second has been established in [8]. We will use the notation that if $X$ is a Banach space then $X^{*}$ is its dual space.

Theorem 1. ([4, Theorem 2.6]) Let $X$ be a reflexive real Banach space, $\Phi: X \longrightarrow \mathbb{R}$ be a coercive continuously Gâteaux differentiable and sequentially weakly lower semicontinuous functional whose Gâteaux derivative admits a con- 
tinuous inverse on $X^{*}, \Psi: X \longrightarrow \mathbb{R}$ be a continuously Gâteaux differentiable functional whose Gâteaux derivative is compact and such that $\Phi(0)=\Psi(0)=0$.

Assume there exist $r>0$ and $\bar{x} \in X$ with $r<\Phi(\bar{x})$ such that

$\left(a_{1}\right) \frac{\sup _{x \in \Phi^{-1}(-\infty, r]} \Psi(x)}{r}<\frac{\Psi(\bar{x})}{\Phi(\bar{x})}$

$\left(a_{2}\right)$ for each $\lambda \in \Lambda_{r}:=\left(\frac{\Phi(\bar{x})}{\Psi(\bar{x})}, \frac{r}{\sup _{x \in \Phi^{-1}(-\infty, r]} \Psi(x)}\right)$, the functional $\Phi-\lambda \Psi$ is coercive.

Then, for each $\lambda \in \Lambda_{r}$, the functional $\Phi-\lambda \Psi$ has at least three distinct critical points in $X$.

Theorem 2. ([8, Corollary 3.1]) Let $X$ be a reflexive real Banach space, $\Phi: X \longrightarrow \mathbb{R}$ be a convex, coercive, and continuously Gâteaux differentiable functional whose Gâteaux derivative admits a continuous inverse on $X^{*}, \Psi$ : $X \longrightarrow \mathbb{R}$ be a continuously Gâteaux differentiable functional whose Gâteaux derivative is compact and such that

$\left(b_{1}\right) \inf _{X} \Phi=\Phi(0)=\Psi(0)=0$.

Assume that there are two positive constants $r_{1}, r_{2}$ and $\bar{x} \in X$, with $2 r_{1}<$ $\Phi(\bar{x})<\frac{r_{2}}{2}$, such that:

$$
\begin{aligned}
& \left(b_{2}\right) \frac{\sup _{x \in \Phi^{-1}\left(-\infty, r_{1}\right)} \Psi(x)}{r_{1}}<\frac{2}{3} \frac{\Psi(\bar{x})}{\Phi(\bar{x})} \\
& \left(b_{3}\right) \frac{\sup _{x \in \Phi^{-1}\left(-\infty, r_{2}\right)} \Psi(x)}{r_{2}}<\frac{1}{3} \frac{\Psi(\bar{x})}{\Phi(\bar{x})}
\end{aligned}
$$

$\left(b_{4}\right)$ For each

$$
\begin{aligned}
\lambda \in \Lambda_{r_{1}, r_{2}}^{\prime}:=\left(\frac{3}{2} \frac{\Phi(\bar{x})}{\Psi(\bar{x})}, \min \left\{\frac{r_{1}}{\sup _{u \in \Phi^{-1}\left(-\infty, r_{1}\right)} \Psi(x)},\right.\right. \\
\left.\left.\frac{\frac{r_{2}}{2}}{\sup _{x \in \Phi^{-1}\left(-\infty, r_{2}\right)} \Psi(x)}\right\}\right)
\end{aligned}
$$

and for every $x_{1}, x_{2} \in X$, which are local minima for the functional $\Phi-\lambda \Psi$ and such that $\Psi\left(x_{1}\right) \geq 0$ and $\Psi\left(x_{2}\right) \geq 0$, we have

$$
\inf _{s \in[0,1]} \Psi\left(s x_{1}+(1-s) x_{2}\right) \geq 0 .
$$


Then, for each $\lambda \in \Lambda_{r_{1}, r_{2}}^{\prime}$, the functional $\Phi-\lambda \Psi$ has at least three distinct critical points that lie in $\Phi^{-1}\left(-\infty, r_{2}\right)$.

We assume that $A$ satisfies the following conditions:

(A1) $A(t)=\left(a_{k l}(t)\right), k=1, \ldots, N, l=1, \ldots, N$, is a symmetric matrix with $a_{k l} \in L^{\infty}[0, T]$ for any $t \in[0, T]$;

(A2) There exists $\kappa>0$ such that $(A(t) x, x) \geq \kappa|x|^{2}$ for any $x \in \mathbb{R}^{N}$ and a.e. $t \in[0, T]$.

Next, we recall some basic concepts. Let

$E=\left\{u:[0, T] \rightarrow \mathbb{R}^{N} \mid u\right.$ is absolutely continuous,

$$
\left.u(0)=u(T), \quad \dot{u} \in L^{2}\left([0, T], \mathbb{R}^{N}\right)\right\}
$$

with the inner product

$$
\prec u, v \succ_{E}=\int_{0}^{T}[(\dot{u}(t), \dot{v}(t))+(u(t), v(t))] d t
$$

where $(\cdot, \cdot)$ denotes the inner product in $\mathbb{R}^{N}$. The corresponding norm is defined by

$$
\|u\|_{E}^{2}=\int_{0}^{T}\left(|\dot{u}(t)|^{2}+|u(t)|^{2}\right) d t \text { for all } u \in E .
$$

For every $u, v \in E$, we define

$$
\prec u, v \succ=\int_{0}^{T}[(\dot{u}(t), \dot{v}(t))+(A(t) u(t), v(t))] d t,
$$

and we observe that, by assumptions (A1) and (A2), this defines an inner product in $E$. Then, $E$ is a separable and reflexive Banach space with the norm

$$
\|u\|=\prec u, u \succ^{\frac{1}{2}} \text { for all } u \in E .
$$

Clearly, $E$ is a uniformly convex Banach space.

A simple computation shows that

$$
(A(t) x, x)=\sum_{k, l=1}^{N} a_{k l}(t) x_{k} x_{l} \leq \sum_{k, l=1}^{N}\left\|a_{k l}\right\|_{\infty}|x|^{2}
$$

for every $t \in[0, T]$ and $x \in \mathbb{R}^{N}$, and this, along with condition (A2), yields

$$
\sqrt{m}\|u\|_{E} \leq\|u\| \leq \sqrt{M}\|u\|_{E}
$$


where $m=\min \{1, \kappa\}$ and $M=\max \left\{1, \sum_{k, l=1}^{N}\left\|a_{k l}\right\|_{\infty}\right\}$. This means that the norms $\|\cdot\|$ and $\|\cdot\|_{E}$ are equivalent.

Since $(E,\|\cdot\|)$ is compactly embedded in $C\left([0, T], \mathbb{R}^{N}\right)$ (see $\left.[29]\right)$, there exists a positive constant $c$ such that

$$
\|u\|_{\infty} \leq c\|u\|
$$

where $\|u\|_{\infty}=\max _{t \in[0, T]}|u(t)|$ and $c=\sqrt{\frac{2}{m}} \max \left\{\frac{1}{\sqrt{T}}, \sqrt{T}\right\}$ (see [12]).

If $u \in E$, then $u$ is absolutely continuous and $\dot{u} \in L^{2}\left([0, T], \mathbb{R}^{N}\right)$. In this case, $\Delta \dot{u}(t)=\dot{u}\left(t^{+}\right)-\dot{u}\left(t^{-}\right)=0$ is not necessarily valid for every $t \in(0, T)$, and the derivative $\dot{u}$ may possess some discontinuities that lead to the impulsive effects.

Next we define what is meant by a solution of (1).

Definition 3. A function $u \in\left\{u \in E: \dot{u} \in\left(W^{1,2}\left(t_{j}, t_{j+1}\right)\right)^{N}, j=\right.$ $0,1,2, \ldots, p\}$ is said to be a classical solution of the problem (1) if $u$ satisfies (1). By a weak solution of problem (1), we mean any $u \in E$ such that

$$
\begin{aligned}
\int_{0}^{T}[(\dot{u}(t), \dot{v}(t))+(A(t) u(t), v(t))-(\nabla H(u(t)), v(t))] d t \\
+\sum_{j=1}^{p} \sum_{i=1}^{N} I_{i j}\left(u_{i}\left(t_{j}\right)\right) v_{i}\left(t_{j}\right)-\lambda \int_{0}^{T}(\nabla F(t, u(t)), v(t)) d t \\
-\mu \int_{0}^{T}(\nabla G(t, u(t)), v(t)) d t=0
\end{aligned}
$$

for every $v \in E$.

The following lemma should come as no surprise.

Lemma 4. ([21, Lemma 2.2]) If $u \in E$ is a weak solution of (1), then $u$ is a classical solution of (1).

We assume throughout that

$$
K:=c^{2}\left(2 L T+\sum_{j=1}^{p} \sum_{i=1}^{N} L_{i j}\right)<1
$$

We set $G^{\theta}:=\int_{[0, T]} \max _{|x| \leq \theta} G(t, x) d t$ for every $\theta>0$ and $G_{\eta}:=\inf _{[0, T] \times[0, \eta]^{N}} G(t, x)$ for every $\eta>0$, where $[0, \eta]^{N}=[0, \eta] \times \ldots \times[0, \eta]$. 


\section{Main Results}

We begin by letting $D>0$ be the constant

$$
D=\frac{\left(T-t_{p}\right)^{2}}{t_{1} t_{p}^{2}}+\frac{t_{1}}{3 t_{p}^{2}}\left(t_{p}^{2}+t_{p} T+T^{2}\right)+\left(t_{p}-t_{1}\right)+\frac{T-t_{p}}{t_{p}^{2}}+\frac{1}{3 t_{p}^{2}}\left(T^{3}-t_{p}^{3}\right) .
$$

For notational purposes, for any two positive constants $\theta$ and $\eta$ such that

$$
\frac{(1+K) D M \eta^{2}}{\int_{t_{1}}^{t_{p}} F(t, \eta \varepsilon) d t}<\frac{(1-K)\left(\frac{\theta}{c}\right)^{2}}{\int_{0}^{T} \max _{|\xi| \leq \theta} F(t, \xi) d t}
$$

where $\varepsilon=(1,0, \cdots, 0) \in \mathbb{R}^{N}$, we take

$$
\lambda \in \Lambda:=\left(\frac{(1+K) D M \eta^{2}}{2 \int_{t_{1}}^{t_{p}} F(t, \eta \varepsilon) d t}, \frac{(1-K)\left(\frac{\theta}{c}\right)^{2}}{2 \int_{0}^{T} \max _{|\xi| \leq \theta} F(t, \xi) d t}\right)
$$

and set

$$
\left.\begin{array}{rl}
\delta_{\lambda, G}=\min \left\{\frac{(1-K)\left(\frac{\theta}{c}\right)^{2}-2 \lambda \int_{0}^{T} \max _{|\xi| \leq \theta} F(t, \xi) d t}{2 G^{\theta}},\right. \\
\\
\frac{(1+K) D M \eta^{2}-2 \lambda \int_{t_{1}}^{t_{p}} F(t, \eta \varepsilon) d t}{2 T G_{\eta}}
\end{array}\right\}
$$

and

$$
\bar{\delta}_{\lambda, G}:=\min \left\{\delta_{\lambda, G}, \frac{1}{\max \left\{0, \frac{2 c^{2}}{(1-K)} \limsup _{|\xi| \rightarrow \infty} \frac{\sup _{t \in[0, T]} G(t, \xi)}{|\xi|^{2}}\right\}}\right\}
$$

We will use the convention that $\rho / 0=+\infty$ for $\rho \in \mathbb{R}^{+}$; hence, $\bar{\delta}_{\lambda, G}=+\infty$ if

$$
\limsup _{|\xi| \rightarrow \infty} \frac{\sup _{t \in[0, T]} G(t, \xi)}{|\xi|^{2}} \leq 0
$$


and $G_{\eta}=G^{\theta}=0$.

We now formulate our main result.

Theorem 5. Assume that there exist two positive constants $\theta$ and $\eta$ with $\frac{\theta}{c \sqrt{D m}}<\eta$ such that

$\left(A_{1}\right) \quad F(t, \xi) \geq 0$ for each $t \in\left[0, t_{1}\right] \cup\left[t_{p}, T\right],|\xi| \leq \frac{\eta T}{t_{p}}$

$\left(A_{2}\right) \quad \frac{\int_{0}^{T} \max _{|\xi| \leq \theta} F(t, \xi) d t}{\theta^{2}}<\frac{1-K}{c^{2}(1+K) D M} \frac{\int_{t_{1}}^{t_{p}} F(t, \eta \varepsilon) d t}{\eta^{2}} ;$

$\left(A_{3}\right) \quad \limsup _{|\xi| \rightarrow \infty} \frac{\sup _{t \in[0, T]} F(t, \xi)}{|\xi|^{2}} \leq 0$.

Then, for each $\lambda \in \Lambda$ and for every function $G:[0, T] \times \mathbb{R}^{N} \rightarrow \mathbb{R}$ that is measurable with respect to $t$ for all $u \in \mathbb{R}^{N}$, continuously differentiable in $u$ for almost every $t \in[0, T]$, and satisfies (2),

$$
G_{\eta} \leq 0, \quad G^{\theta} \geq 0
$$

and

$$
\limsup _{|\xi| \rightarrow \infty} \frac{\sup _{t \in[0, T]} G(t, \xi)}{|\xi|^{2}}<+\infty
$$

there exists $\bar{\delta}_{\lambda, G}>0$ given by (7) such that, for each $\mu \in\left[0, \bar{\delta}_{\lambda, G}\right)$, the problem (1) admits at least three distinct classical periodic solutions in $E$.

Proof. Fix $\lambda$ and $\mu$ as in the conclusion of the theorem. Set $X=E$ and define the functionals $\Phi, \Psi: X \rightarrow \mathbb{R}$ by

$$
\Phi(u)=\frac{1}{2}\|u\|^{2}+\sum_{j=1}^{p} \sum_{i=1}^{N} \int_{0}^{u_{i}\left(t_{j}\right)} I_{i j}(s) d s-\int_{0}^{T} H(u(t)) d t
$$

and

$$
\Psi(u)=\int_{0}^{T}\left[F(t, u(t))+\frac{\mu}{\lambda} G(t, u(t))\right] d t
$$

for every $u \in X$. It is well known that $\Psi$ is a Gâteaux differentiable functional whose Gâteaux derivative at the point $u \in X$ is the functional $\Psi^{\prime}(u) \in X^{*}$ given by

$$
\Psi^{\prime}(u) v=\int_{0}^{T}\left(\nabla F(t, u(t))+\frac{\mu}{\lambda} \nabla G(t, u(t)), v(t)\right) d t
$$


for every $v \in X$, and $\Psi^{\prime}: X \rightarrow X^{*}$ is a compact operator. Moreover, $\Phi$ is a Gâteaux differentiable functional whose Gâteaux derivative at the point $u \in X$ is the functional $\Phi^{\prime}(u) \in X^{*}$ given by

$$
\begin{aligned}
\Phi^{\prime}(u) v= & \int_{0}^{T}[(\dot{u}(t), \dot{v}(t))+(A(t) u(t), v(t))-(\nabla H(u(t)), v(t))] d t \\
& +\sum_{j=1}^{p} \sum_{i=1}^{N} I_{i j}\left(u_{i}\left(t_{j}\right)\right) v_{i}\left(t_{j}\right)
\end{aligned}
$$

for every $v \in X$. Also, [22, Proposition 2.4] ensures that $\Phi^{\prime}$ admits a continuous inverse on $X^{*}$.

To show that $\Phi$ is sequentially weakly lower semicontinuous, let $u_{n} \in X$ with $u_{n} \rightarrow u$ weakly in $X$. We then have $\liminf _{n \rightarrow+\infty}\left\|u_{n}\right\| \geq\|u\|$ and $u_{n} \rightarrow u$ uniformly on $[0, T]$. Since $H$ is continuous,

$$
\begin{aligned}
\liminf _{n \rightarrow+\infty}( & \left.\frac{1}{2}\left\|u_{n}\right\|^{2}+\sum_{j=1}^{p} \sum_{i=1}^{N} \int_{0}^{u_{n i}\left(t_{j}\right)} I_{i j}(s) d s-\int_{0}^{T} H\left(u_{n}(t)\right) d t\right) \\
\geq & \frac{1}{2}\|u\|^{2}+\sum_{j=1}^{p} \sum_{i=1}^{N} \int_{0}^{u_{n i}\left(t_{j}\right)} I_{i j}(s) d s-\int_{0}^{T} H(u(t)) d t .
\end{aligned}
$$

That is, $\liminf _{n \rightarrow+\infty} \Phi\left(u_{n}\right) \geq \Phi(u)$, which means that $\Phi$ is sequentially weakly lower semicontinuous.

From (3) and the fact that $H(0, \ldots, 0)=0$, we have $|H(\xi)| \leq L|\xi|^{2}$ for all $\xi \in \mathbb{R}^{N}$. This, in conjunction with the fact that $-L_{i j}|s|^{2} \leq I_{i j}(s) s \leq L_{i j}|s|^{2}$ for every $s \in \mathbb{R}$ for all $i=1,2, \ldots, N, j=1,2, \ldots, p$, and inequality (5), we have

$$
\frac{1}{2}(1-K)\|u\|^{2} \leq \Phi(u) \leq \frac{1}{2}(1+K)\|u\|^{2}
$$

for $u \in X$. Let $r=\frac{1}{2}(1-K)\left(\frac{\theta}{c}\right)^{2}$ and

$$
w(t)= \begin{cases}\left(T+\frac{t_{p}-T}{t_{1}} t\right) \frac{\eta \varepsilon}{t_{p}}, & t \in\left[0, t_{1}\right), \\ \eta \varepsilon, & t \in\left[t_{1}, t_{p}\right], \\ \frac{\eta \varepsilon}{t_{p}} t, & t \in\left(t_{p}, T\right] .\end{cases}
$$

It is easy to see that $w \in X=E$ and $\|w\|_{E}^{2}=D \eta^{2}$. Hence, in view of (4),

$$
D m \eta^{2} \leq\|w\|^{2} \leq D M \eta^{2},
$$


and this together with the condition $\frac{\theta}{c \sqrt{D m}}<\eta$, ensures that $0<r<\Phi(w)$. From (5) and (10), we see that for each $u \in X$,

$$
\begin{aligned}
\Phi^{-1}(-\infty, r] & =\{u \in X: \Phi(u) \leq r\} \\
& \subseteq\left\{u \in X: \frac{1}{2}(1-K)\|u\|^{2} \leq r\right\} \\
& \subseteq\{u \in X:|u(t)| \leq \theta \text { for each } t \in[0, T]\}
\end{aligned}
$$

and it follows that

$$
\begin{aligned}
\sup _{u \in \Phi^{-1}(-\infty, r]} \Psi(u) & =\sup _{u \in \Phi^{-1}(-\infty, r]} \int_{0}^{T}\left[F(t, u(t))+\frac{\mu}{\lambda} G(t, u(t))\right] d t \\
& \leq \int_{0}^{T} \sup _{|\xi| \leq \theta} F(t, \xi) d x+\frac{\mu}{\lambda} G^{\theta} .
\end{aligned}
$$

On the other hand, from condition $\left(A_{1}\right)$, we have

$$
\begin{aligned}
\Psi(w) & \geq \int_{t_{1}}^{t_{p}} F(t, \eta \varepsilon) d t+\frac{\mu}{\lambda} \int_{0}^{T} G(t, w(t)) d t \\
& \geq \int_{t_{1}}^{t_{p}} F(t, \eta \varepsilon) d t+T \frac{\mu}{\lambda} \inf _{[0, T] \times[0, \eta]^{N}} G \\
& =\int_{t_{1}}^{t_{p}} F(t, \eta \varepsilon) d t+T \frac{\mu}{\lambda} G_{\eta} .
\end{aligned}
$$

Therefore, we have

$$
\begin{aligned}
\sup _{u \in \Phi^{-1}(-\infty, r]} \Psi(u) & =\frac{\sup _{u \in \Phi^{-1}(-\infty, r)} \int_{0}^{T}\left[F(t, u(t))+\frac{\mu}{\lambda} G(t, u(t))\right] d t}{r} \\
\leq & \frac{\int_{0}^{T} \sup _{|\xi| \leq \theta} F(t, \xi) d x+\frac{\mu}{\lambda} G^{\theta}}{\frac{1}{2}(1-K)\left(\frac{\theta}{c}\right)^{2}},
\end{aligned}
$$

and so

$$
\frac{\Psi(w)}{\Phi(w)} \geq \frac{\int_{t_{1}}^{t_{p}} F(t, \eta \varepsilon) d t+\frac{\mu}{\lambda} \int_{0}^{T} G(t, w(t)) d t}{\frac{1}{2}(1+K) D M \eta^{2}}
$$




$$
\geq \frac{\int_{t_{1}}^{t_{p}} F(t, \eta \varepsilon) d t+T \frac{\mu}{\lambda} G_{\eta}}{\frac{1}{2}(1+K) D M \eta^{2}}
$$

Since $\mu<\delta_{\lambda, G}$, we have

$$
\mu<\frac{(1-K)\left(\frac{\theta}{c}\right)^{2}-2 \lambda \int_{0}^{T} \max _{|\xi| \leq \theta} F(t, \xi) d t}{2 G^{\theta}},
$$

which implies

$$
\frac{\int_{0}^{T} \max _{|\xi| \leq \theta} F(t, \xi) d t+\frac{\mu}{\lambda} G^{\theta}}{\frac{1}{2}(1-K)\left(\frac{\theta}{c}\right)^{2}}<\frac{1}{\lambda}
$$

Moreover, since

$$
\mu<\frac{(1+K) D M \eta^{2}-2 \lambda \int_{t_{1}}^{t_{p}} F(t, \eta \varepsilon) d t}{2 T G_{\eta}}
$$

and $G_{\eta} \leq 0$, we see that

$$
\frac{\int_{t_{1}}^{t_{p}} F(t, \eta \varepsilon) d t+T \frac{\mu}{\lambda} G_{\eta}}{\frac{1}{2}(1+K) D M \eta^{2}}>\frac{1}{\lambda} .
$$

Therefore,

$$
\frac{\int_{0}^{T} \max _{|\xi| \leq \theta} F(t, \xi) d t+\frac{\mu}{\lambda} G^{\theta}}{\frac{1}{2}(1-K)\left(\frac{\theta}{c}\right)^{2}}<\frac{1}{\lambda}<\frac{\int_{t_{1}}^{t_{p}} F(t, \eta \varepsilon) d t+T \frac{\mu}{\lambda} G_{\eta}}{\frac{1}{2}(1+K) D M \eta^{2}} .
$$

Hence, from (13)-(15), we see that condition $\left(a_{1}\right)$ of Theorem 1 is satisfied.

Finally, since $\mu<\bar{\delta}_{\lambda, G}$, by (9), we can fix $l>0$ such that

$$
\limsup _{|\xi| \rightarrow \infty} \frac{\sup _{t \in[0, T]} G(t, \xi)}{|\xi|^{2}}<l
$$


and $\mu l<\frac{1-K}{2 T c^{2}}$. Therefore, there exists a function $h \in L^{1}([0, T])$ such that

$$
G(t, \xi) \leq l|\xi|^{2}+h(t)
$$

for every $t \in[0, T]$ and $\xi \in \mathbb{R}^{N}$. Now, for $\lambda>0$, choose $0<\epsilon<\frac{1-K}{2 \lambda T c^{2}}-\frac{\mu l}{\lambda}$. From $\left(A_{3}\right)$, there is a function $h_{\epsilon} \in L^{1}([0, T])$ such that

$$
F(t, \xi) \leq \epsilon|\xi|^{2}+h_{\epsilon}(t)
$$

for every $t \in[0, T]$ and $\xi \in \mathbb{R}^{N}$. From (5) (10), (16), and (17), it follows that, for each $u \in X$,

$$
\begin{aligned}
\Phi(u)-\lambda \Psi(u)= & \frac{1}{2}\|u\|^{2}+\sum_{j=1}^{p} \sum_{i=1}^{N} \int_{0}^{u_{i}\left(t_{j}\right)} I_{i j}(s) d s-\int_{0}^{T} H(u(t)) d t \\
& -\lambda \int_{0}^{T}\left[F(t, u(t))+\frac{\mu}{\lambda} G(t, u(t))\right] d t \\
\geq & \frac{1}{2}(1-K)\|u\|^{2}-\lambda \epsilon \int_{0}^{T}|u(t)|^{2} d t-\lambda\left\|h_{\epsilon}\right\|_{1} \\
& -\mu l \int_{0}^{T}|u(t)|^{2} d t-\mu\|h\|_{1} \\
\geq & \left(\frac{1}{2}(1-K)-\lambda \epsilon T c^{2}-\mu l T c^{2}\right)\|u\|^{2}-\lambda\left\|h_{\epsilon}\right\|_{1}-\mu\|h\|_{1},
\end{aligned}
$$

and so

$$
\lim _{\|u\| \rightarrow+\infty}(\Phi(u)-\lambda \Psi(u))=+\infty,
$$

which means the functional $\Phi-\lambda \Psi$ is coercive. Now (13)-(15) imply

$$
\lambda \in\left(\frac{\Phi(w)}{\Psi(w)}, \frac{r}{\sup _{\Phi(x) \leq r} \Psi(x)}\right),
$$

so condition $\left(a_{2}\right)$ of Theorem 1 is satisfied. Clearly, weak solutions of problem (1) are precisely the solutions of the equation $\Phi^{\prime}(u)-\lambda \Psi^{\prime}(u)=0$. Therefore, in view of Lemma 4, the conclusion of the theorem follows from Theorem 1 with $\bar{x}=w$.

Next, we present a variant of Theorem 5 in which no asymptotic condition on the nonlinear term $G$ is required, but $F$ and $G$ are assumed to be nonnegative. 
For positive constants $\theta_{1}, \theta_{2}$, and $\eta$ with

$$
\begin{aligned}
& \frac{3}{2} \frac{(1+K) D M \eta^{2}}{\int_{t_{1}}^{t_{p}} F(t, \eta \varepsilon) d t} \\
& \quad<(1-K)\left(\frac{1}{c}\right)^{2} \min \left\{\frac{\theta_{1}^{2}}{\int_{0}^{T} \max _{|\xi| \leq \theta_{1}} F(t, \xi) d t}, \frac{\theta_{2}^{2}}{2 \int_{0}^{T} \max _{|\xi| \leq \theta_{2}} F(t, \xi) d t}\right\},
\end{aligned}
$$

we introduce the notation

$$
\begin{aligned}
\Lambda^{\prime}:= & \frac{3}{4} \frac{(1+K) D M \eta^{2}}{\int_{t_{1}}^{t_{p}} F(t, \eta \varepsilon) d t}, \\
& \left.\frac{1}{2}(1-K)\left(\frac{1}{c}\right)^{2} \min \left\{\frac{\theta_{1}^{2}}{\int_{0}^{T} \max _{|\xi| \leq \theta_{1}} F(t, \xi) d t}, \frac{\theta_{2}^{2}}{2 \int_{0}^{T} \max _{|\xi| \leq \theta_{2}} F(t, \xi) d t}\right\}\right)
\end{aligned}
$$

We then have the following existence result.

Theorem 6. Let $F:[0, T] \times \mathbb{R}^{N} \rightarrow \mathbb{R}$ be a non-negative function. Assume that there exist three positive constants $\theta_{1}, \theta_{2}$, and $\eta$ with

$$
\frac{\theta_{1}}{c} \sqrt{\frac{2}{D m}}<\eta<\sqrt{\frac{1-K}{2 D M(1+K)}} \frac{\theta_{2}}{c}
$$

such that condition $\left(A_{1}\right)$ in Theorem 5 holds. In addition, assume that $\left(B_{1}\right)$

$$
\begin{array}{r}
\max \left\{\frac{\int_{0}^{T} \max _{|\xi| \leq \theta_{1}} F(t, \xi) d t}{\theta_{1}^{2}}, \frac{2 \int_{0}^{T} \max _{|\xi| \leq \theta_{2}} F(t, \xi) d t}{\theta_{2}^{2}}\right\} \\
<\frac{2}{3} \frac{1-K}{c^{2}(1+K) D M} \frac{\int_{t_{1}}^{t_{p}} F(t, \eta \varepsilon) d t}{\eta^{2}} .
\end{array}
$$


Then, for each $\lambda \in \Lambda^{\prime}$ and for every nonnegative function $G:[0, T] \times \mathbb{R}^{N} \rightarrow \mathbb{R}$ that is measurable with respect to $t$ for all $u \in \mathbb{R}^{N}$, continuously differentiable in $u$ for almost every $t \in[0, T]$, and satisfies (2), there exists $\delta_{\lambda, G}^{*}>0$ given by

$$
\begin{aligned}
\min \left\{\frac{(1-K)\left(\frac{\theta_{1}}{c}\right)^{2}-2 \lambda \int_{0}^{T} \max _{|\xi| \leq \theta} F(t, \xi) d t}{2 G^{\theta_{1}}},\right. \\
\\
\left.\quad \frac{(1-K)\left(\frac{\theta_{2}}{c}\right)^{2}-4 \lambda \int_{0}^{T} \max _{|\xi| \leq \theta} F(t, \xi) d t}{4 G^{\theta_{2}}}\right\}
\end{aligned}
$$

such that, for each $\mu \in\left[0, \delta_{\lambda, G}^{*}\right)$, the problem (1) admits at least three distinct classical periodic solutions $u^{k}=\left(u_{1}^{k}, \ldots, u_{N}^{k}\right)$ for $k=1,2,3$, such that

$$
\left|u^{k}(t)\right|<\theta_{2} \quad \text { for all } t \in[0, T], k=1,2,3 \text {. }
$$

Proof. Fix $\lambda, G$, and $\mu$ as in the conclusion of the theorem and take $X, \Phi$, and $\Psi$ as in the proof of Theorem 5. Note that the regularity assumptions in Theorem 2 on $\Phi$ and $\Psi$, and condition $\left(b_{1}\right)$ are satisfied. We need to show that $\left(b_{2}\right)$ and $\left(b_{3}\right)$ hold, so choose $w$ as in (11) and set

$$
r_{1}=\frac{1}{2}(1-K)\left(\frac{\theta_{1}}{c}\right)^{2} \quad \text { and } \quad r_{2}=\frac{1}{2}(1-K)\left(\frac{\theta_{2}}{c}\right)^{2} .
$$

From the condition $\frac{\theta_{1}}{c} \sqrt{\frac{2}{D m}}<\eta<\frac{\theta_{2}}{c} \sqrt{\frac{1-K}{2 D M(1+K)}}$, and recalling (10), we see that $2 r_{1}<\Phi(w)<\frac{r_{2}}{2}$. Since $\mu<\delta_{\lambda, G}^{*}$ and $G_{\eta}=0$, we have

$$
\begin{aligned}
\frac{\sup _{u \in \Phi^{-1}\left(-\infty, r_{1}\right)} \Psi(u)}{r_{1}} & =\frac{\sup _{u \in \Phi^{-1}\left(-\infty, r_{1}\right)} \int_{0}^{T}\left[F(t, u(t))+\frac{\mu}{\lambda} G(t, u(t))\right] d t}{r_{1}} \\
\leq & \frac{\int_{0}^{T} \sup _{|\xi| \leq \theta_{1}} F(t, \xi) d t+\frac{\mu}{\lambda} G^{\theta_{1}}}{\frac{1}{2}(1-K)\left(\frac{\theta_{1}}{c}\right)^{2}}
\end{aligned}
$$




$$
\begin{aligned}
& <\frac{1}{\lambda}<\frac{2}{3} \frac{\int_{t_{1}}^{t_{p}} F(t, \eta \varepsilon) d t+T \frac{\mu}{\lambda} G \eta}{\frac{1}{2}(1+K) D M \eta^{2}} \\
& \leq \frac{2}{3} \frac{\Psi(w)}{\Phi(w)}
\end{aligned}
$$

and

$$
\begin{aligned}
\frac{2 \sup _{u \in \Phi^{-1}\left(-\infty, r_{2}\right)} \Psi(u)}{r_{2}} & =\frac{2 \sup _{u \in \Phi^{-1}\left(-\infty, r_{2}\right)} \int_{0}^{T}\left[F(t, u(t))+\frac{\mu}{\lambda} G(t, u(t))\right] d t}{r_{2}} \\
& \leq \frac{2 \int_{0}^{T} \sup _{|t| \leq \theta_{2}} F(t, \xi) d x+2 \frac{\mu}{\lambda} G^{\theta_{2}}}{\frac{1}{2}(1-K)\left(\frac{\theta_{2}}{c}\right)^{2}} \\
& <\frac{1}{\lambda}<\frac{2}{3} \frac{\int_{t_{1}}^{t_{p}} F(t, \eta \varepsilon) d t+T \frac{\mu}{\lambda} G_{\eta}}{\frac{1}{2}(1+K) D M \eta^{2}} \\
& \leq \frac{2}{3} \frac{\Psi(w)}{\Phi(w)}
\end{aligned}
$$

Therefore, $\left(b_{2}\right)$ and $\left(b_{3}\right)$ of Theorem 2 are satisfied.

To complete our proof, we need to show that condition $\left(b_{4}\right)$ is satisfied. Let $u^{*}=\left(u_{1}^{\star}, \ldots, u_{N}^{\star}\right)$ and $u^{\star \star}=\left(u_{1}^{\star \star}, \ldots, u_{N}^{\star \star}\right)$ be two local minima for $\Phi-\lambda \Psi$. Then $u^{\star}$ and $u^{\star \star}$ are critical points for $\Phi-\lambda \Psi$, and so, they are weak solutions for the problem (1). Since $F$ and $G$ are nonnegative, $F\left(t, s u^{\star}+(1-s) u^{\star \star}\right) \geq 0$ and $G\left(t, s u^{\star}+(1-s) u^{\star \star}\right) \geq 0$, and so $\Psi\left(s u^{\star}+(1-s) u^{\star \star}\right) \geq 0$ for all $s \in[0,1]$. Hence, by Theorem 2, for every

$$
\lambda \in\left\{\frac{3}{2} \frac{\Phi(w)}{\Psi(w)}, \min \left\{\frac{r_{1}}{\sup _{u \in \Phi^{-1}\left(\left(-\infty, r_{1}\right)\right)} \Psi(u)}, \frac{r_{2} / 2}{\sup _{u \in \Phi^{-1}\left(\left(-\infty, r_{2}\right)\right)} \Psi(u)}\right\}\right),
$$

the functional $\Phi-\lambda \Psi$ has at least three distinct critical points that are weak solutions of problem (1). An application of Lemma 4 completes the proof of the theorem.

Before presenting our next theorem, note that if $G$ is independent of $t$, i.e., $G(t, x)=\bar{G}(x)$, then $\bar{G}^{\theta}=T \max _{|x| \leq \theta} \bar{G}(x)$ and $\bar{G}_{\eta}=\inf _{[0, \eta]^{N}} \bar{G}(x)$. The following result is a special case of Theorem 5 . 
Theorem 7. Let $F: \mathbb{R}^{N} \rightarrow \mathbb{R}$ be a continuously differentiable function such that

$$
\liminf _{x \rightarrow 0} \frac{\max _{|\xi| \leq x} F(\xi)}{|x|^{2}}=\limsup _{|\xi| \rightarrow+\infty} \frac{F(\xi)}{|\xi|^{2}}=0 .
$$

Then, there is $\lambda^{*}>0$ such that for each $\lambda>\lambda^{*}$ and for every continuously differentiable function $G: \mathbb{R}^{N} \rightarrow \mathbb{R}$ satisfying $\bar{G}_{\eta} \leq 0, \bar{G}^{\theta} \geq 0$, and the asymptotic condition

$$
\limsup _{|\xi| \rightarrow \infty} \frac{G(\xi)}{|\xi|^{2}}<+\infty
$$

there exists $\delta_{\lambda, G}^{*}>0$ such that, for each $\mu \in\left[0, \delta_{\lambda, G}^{*}\right)$, the problem

$$
\left\{\begin{array}{l}
-\ddot{u}(t)+A(t) u(t)=\lambda \nabla F(u(t))+\mu \nabla G(u(t)) \\
+\nabla H(u(t)), \quad \text { a.e. } t \in[0, T] \\
\Delta\left(\dot{u}_{i}\left(t_{j}\right)\right)=I_{i j}\left(u_{i}\left(t_{j}\right)\right), \quad i=1,2, \ldots, N, j=1,2, \ldots, p \\
u(0)-u(T)=\dot{u}(0)-\dot{u}(T)=0
\end{array}\right.
$$

admits at least three classical periodic solutions.

Proof. Fix $\lambda>\lambda^{*}:=\frac{(1+K) D M \eta^{2}}{2\left(t_{p}-t_{1}\right) F(\eta \varepsilon)}$ for some $\eta>0$. Since

$$
\liminf _{x \rightarrow 0} \frac{\max _{|\xi| \leq x} F(\xi)}{x^{2}}=0
$$

there is a sequence $\left\{\theta_{n}\right\} \subset(0,+\infty)$ such that $\lim _{n \rightarrow \infty} \theta_{n}=0$ and

$$
\lim _{n \rightarrow \infty} \frac{\max _{|\xi| \leq \theta_{n}} F(\xi)}{\theta_{n}^{2}}=0
$$

Hence, there exists $\bar{\theta}>0$ such that

$$
\frac{\max _{|\xi| \leq \bar{\theta}} F(\xi)}{\bar{\theta}^{2}}<\min \left\{\frac{(1-K)\left(t_{p}-t_{1}\right) F(\eta \varepsilon)}{c^{2} T(1+K) D M \eta^{2}}, \frac{1-K}{2 \lambda T c^{2}}\right\}
$$

and $\frac{\bar{\theta}}{c \sqrt{D m}}<\eta$. The conclusion follows from Theorem 5 .

The following result is a consequence of Theorem 6 . 
Theorem 8. Let $F: \mathbb{R}^{2} \rightarrow \mathbb{R}$ be a nonnegative continuously differentiable function such that

$$
\lim _{x \rightarrow 0^{+}} \frac{\max _{\left|\left(\xi_{1}, \xi_{2}\right)\right| \leq x} F\left(\xi_{1}, \xi_{2}\right)}{x^{2}}=0
$$

and

$$
\max _{\left|\left(\xi_{1}, \xi_{2}\right)\right| \leq 4} F\left(\xi_{1}, \xi_{2}\right)<\frac{4\left(1-36 L-6 \sum_{j=1}^{2} \sum_{i=1}^{2} L_{i j}\right)}{378\left(1+36 L+6 \sum_{j=1}^{2} \sum_{i=1}^{2} L_{i j}\right)} F(2,0) .
$$

Then, for every

$$
\begin{gathered}
\lambda \in\left(\frac{21\left(1+36 L+6 \sum_{j=1}^{2} \sum_{i=1}^{2} L_{i j}\right)}{F(2,0)},\right. \\
\left.\frac{4\left(1-36 L-6 \sum_{j=1}^{2} \sum_{i=1}^{2} L_{i j}\right)}{18 \max _{\left|\left(\xi_{1}, \xi_{2}\right)\right| \leq 4} F\left(\xi_{1}, \xi_{2}\right)}\right)
\end{gathered}
$$

and for every nonnegative continuously differentiable function $G: \mathbb{R}^{2} \rightarrow \mathbb{R}$, there exists $\delta_{\lambda, G}^{*}>0$ such that, for each $\mu \in\left[0, \delta_{\lambda, G}^{*}\right)$, the problem

$$
\left\{\begin{array}{l}
-\ddot{u}(t)+A(t) u(t)=\lambda \nabla F(t, u(t)) \\
\quad+\mu \nabla G(t, u(t))+\nabla H(u(t)), \text { a.e. } t \in[0,3] \\
\Delta\left(\dot{u}_{i}\left(t_{j}\right)\right)=I_{i j}\left(u_{i}\left(t_{j}\right)\right), \quad i=1,2, t_{1}=1, t_{2}=2 \\
u(0)-u(T)=\dot{u}(0)-\dot{u}(T)=0
\end{array}\right.
$$

where $A(t)$ is the second-order identity matrix, admits at least three classical periodic solutions.

Proof. Here $N=p=2, T=3, t_{1}=1, t_{2}=2, \theta_{2}=4$, and $\eta=2$. Therefore, since $m=1, M=2, c=\sqrt{6}, D=\frac{7}{2}$, we see that

$$
\frac{3}{2} \frac{\frac{1}{2}(1+K) D M \eta^{2}}{\int_{t_{1}}^{t_{p}} F(t, \eta \varepsilon) d t}=\frac{21\left(1+36 L+6 \sum_{j=1}^{2} \sum_{i=1}^{2} L_{i j}\right)}{F(2,0)}
$$

and

$$
\frac{1}{2}(1-K)\left(\frac{1}{c}\right)^{2} \frac{\theta_{2}^{p}}{2 \int_{0}^{T} \max _{|\xi| \leq \theta_{2}} F(t, \xi) d t}=\frac{4\left(1-36 L-6 \sum_{j=1}^{2} \sum_{i=1}^{2} L_{i j}\right)}{18 \max _{\left|\left(\xi_{1}, \xi_{2}\right)\right| \leq 4} F\left(\xi_{1}, \xi_{2}\right)} .
$$


Moreover, since

$$
\lim _{x \rightarrow 0^{+}} \frac{\max _{\left|\left(\xi_{1}, \xi_{2}\right)\right| \leq x} F\left(\xi_{1}, \xi_{2}\right)}{x^{2}}=0,
$$

there exists a positive constant $\theta_{1}<\sqrt{42}$ such that

$$
\frac{\max _{\left|\left(\xi_{1}, \xi_{2}\right)\right| \leq \theta_{1}} F\left(\xi_{1}, \xi_{2}\right)}{\theta_{1}^{2}}<\frac{1-36 L-6 \sum_{j=1}^{2} \sum_{i=1}^{2} L_{i j}}{378\left(1+36 L+6 \sum_{j=1}^{2} \sum_{i=1}^{2} L_{i j}\right)} F(2,0)
$$

and

$$
\frac{\theta_{1}^{2}}{\max _{\left|\left(\xi_{1}, \xi_{2}\right)\right| \leq \theta_{1}} F\left(\xi_{1}, \xi_{2}\right)}>\frac{8}{\max _{\left|\left(\xi_{1}, \xi_{2}\right)\right| \leq 4} F\left(\xi_{1}, \xi_{2}\right)} .
$$

Now it is easy to see that all assumptions of Theorem 6 are satisfied, and so the conclusion follows.

\section{References}

[1] L. Bai, B. Dai, Application of variational method to a class of Dirichlet boundary value problems with impulsive effects, J. Franklin Ins. 348 (2011), 2607-2624.

[2] D. Bainov, P. Simeonov, Systems with Impulse Effect, Ellis Horwood Series: Mathematics and Its Applications, Ellis Horwood, Chichester, 1989.

[3] M. Benchohra, J. Henderson, S. Ntouyas, Theory of Impulsive Differential Equations, Contemporary Mathematics and Its Applications, 2, Hindawi Publishing Corporation, New York, (2006).

[4] G. Bonanno, P. Candito, Non-differentiable functionals and applications to elliptic problems with discontinuous nonlinearities, J. Differential Equations 244 (2008), 3031-3059.

[5] G. Bonanno, A. Chinnì, Existence of three solutions for a perturbed two-point boundary value problem, Appl. Math. Lett. 23 (2010), 807-811.

[6] G. Bonanno, G. D'Aguì, Multiplicity results for a perturbed elliptic Neumann problem, Abstr. Appl. Anal. 2010 (2010), doi:10.1155/2010/564363, 10 pages.

[7] G. Bonanno, B. Di Bella, J. Henderson, Existence of solutions to second-order boundaryvalue problems with small perturbations of impulses, Electron. J. Differ. Eqs. 2013 (2013), No. 126, pp. 1-14.

[8] G. Bonanno, S. A. Marano, On the structure of the critical set of non-differentiable functions with a weak compactness condition, Appl. Anal. 89 (2010), 1-10.

[9] G. Bonanno, R. Livrea, Existence and multiplicity of periodic solutions for second order Hamiltonian systems depending on a parameter, J. Convex Anal. 20 (2013), 1075-1094.

[10] G. Bonanno, R. Livrea, Multiple periodic solutions for Hamiltonian systems with not coercive potential, J. Math. Anal. Appl. 363 (2010), 627-638.

[11] G. Bonanno, R. Livrea, Periodic solutions for a class of second-order Hamiltonian systems, Electron. J. Differ. Eqs. 2005 (2005), No. 115, pp. 1-13. 
[12] H. Chen, Z. He, New results for perturbed Hamiltonian systems with impulses, Appl. Math. Comput. 218 (2012), 9489-9497.

[13] G. Chen, S. Ma, Periodic solutions for Hamiltonian systems without AmbrosettiRabinowitz condition and spectrum 0, J. Math. Anal. Appl. 379 (2011), 842-851.

[14] G. Cordaro, Three periodic solutions to an eigenvalue problem for a class of second order Hamiltonian systems, Abstr. Appl. Anal. 18 (2003), 1037-1045.

[15] G. Cordaro, G. Rao, Three periodic solutions for perturbed second order Hamiltonian systems, J. Math. Anal. Appl. 359 (2009), 780-785.

[16] V. Coti-Zelati, I. Ekeland, E. Sere, A variational approach to homoclinic orbits in Hamiltonian systems, Math. Ann. 288 (1990), 133-160.

[17] G. D'Agui, S. Heidarkhani, G. Molica Bisci, Multiple solutions for a perturbed mixed boundary value problem involving the one-dimensional p-Laplacian, Electron. J. Qual. Theory Diff. Eqns. 2013 (2013), No. 24, 1-14.

[18] Y. Ding, C. Lee, Periodic solutions for Hamiltonian systems, SIAM J. Math. Anal. 32 (2000), 555-571.

[19] F. Faraci, Multiple periodic solutions for second order systems with changing sign potential, J. Math. Anal. Appl. 319 (2006), 567-578.

[20] M. Ferrara, S. Heidarkhani, Multiple solutions for perturbed p-Laplacian boundary value problem with impulsive effects, Electron. J. Differ. Eqs. 2014 (2014), No. 106, pp. 1-14.

[21] J. R. Graef, S. Heidarkhani, L. Kong, Infinitely many periodic solutions to a class of perturbed second-order impulsive Hamiltonian systems, preprint.

[22] J. R. Graef, S. Heidarkhani, L. Kong, Nontrivial periodic solutions to second-order impulsive Hamiltonian systems, Electron. J. Differential Equations 2015 (2015), No. 204, pp. $1-17$.

[23] J. R. Graef, J. Henderson, A. Ouahab, Impulsive Differential Inclusions, A Fixed Point Approach, De Gruyter Series in Nonlinear Analysis and Applications, Vol. 20, De Gruyter, Berlin, 2013.

[24] H. Gu, T. An, Existence of infinitely many periodic solutions for second-order Hamiltonian systems, Electron. J. Differ. Eqs. 2013 (2013), No. 251, pp. 1-10.

[25] X. He, X. Wu, Periodic solutions for a class of nonautonomous second-order Hamiltonian systems, J. Math. Anal. Appl. 341 (2008), 1354-1364.

[26] M. Izydorek, J. Janczewska, Homoclinic solutions for a class of second order Hamiltonian systems, J. Differential Equations 219 (2005), 375-389.

[27] V. Lakshmikantham, D. D. Bainov, P. S. Simeonov, Theory of Impulsive Differential Equations, World Scientific, Singapore, 1989.

[28] Y. Long, Nonlinear oscillations for classical Hamiltonian systems with bi-even subquadratic potentials, Nonlinear Anal. 25 (1995), 1665-1671.

[29] J. Mawhin, M. Willem, Critical Point Theory and Hamiltonian Systems, Springer, New York, 1989.

[30] J. J. Nieto, D. O'Regan, Variational approach to impulsive differential equations, Nonlinear Anal. RWA 10 (2009), 680-690. 
[31] P. H. Rabinowitz, Homoclinic orbits for a class of Hamiltonian systems, Proc. Roy. Soc. Edinb. 114 (1990), 33-38.

[32] P. H. Rabinowitz, Variational methods for Hamiltonian systems, in: Handbook of Dynamical Systems, vol. 1, North-Holland, 2002, Part 1, Chapter 14, pp. 1091-1127.

[33] A. M. Samoilenko, N. A. Perestyuk, Impulsive Differential Equations, World Scientific, Singapore, 1995.

[34] J. Sun, H. Chen, J. J. Nieto, Infinitely many solutions for second-order Hamiltonian system with impulsive effects, Math. Comput. Model. 54 (2011), 544-555.

[35] J. Sun, H. Chen, J. J. Nieto, M. Otero-Novoa, The multiplicity of solutions for perturbed second-order Hamiltonian systems with impulsive effects, Nonlinear Anal. 72 (2010), $4575-4586$.

[36] H. Suo, L. Di, Y. An, C. Chu, Existence and multiplicity of periodic solutions for some second-order Hamiltonian systems, J. Inequalities Appl. 2014, 2014:411.

[37] C. Tang, Periodic solutions for nonautonomous second order systems with sublinear nonlinearity, Proc. Amer. Math. Soc. 126 (1998), 3263-3270.

[38] C.-L. Tang, X.-P. Wu, Periodic solutions for a class of nonautonomous subquadratic second order Hamiltonian systems, J. Math. Anal. Appl. 275 (2002), 870-882.

[39] Y. Tian, W. Ge, Applications of variational methods to boundary-value problem for impulsive differential equations, Proc. Edinb. Math. Soc. 51 (2008), 509-527.

[40] Z. Wang, J. Zhang, Periodic solutions of a class of second order non-autonomous Hamiltonian systems, Nonlinear Anal. 72 (2010), 4480-4487.

[41] D. Zhang, B. Dai, Existence of solutions for nonlinear impulsive differential equations with Dirichlet boundary conditions, Math. Comput. Modelling 53 (2011), 1154-1161.

[42] Q. Zhang, C. Liu, Infinitely many periodic solutions for second order Hamiltonian systems, J. Differential Equations 251 (2011), 816-833.

[43] Q. Zhang, X. Tang, New existence of periodic solutions for second order non-autonomous Hamiltonian systems, J. Math. Anal. Appl. 369 (2010), 357-367.

[44] J. Zhou, Y. Li, Existence of solutions for a class of second order Hamiltonian systems with impulsive effects, Nonlinear Anal. 72 (2010), 1594-1603.

[45] X. Zhang, Y. Zhou, Periodic solutions of non-autonomous second order Hamiltonian systems, J. Math. Anal. Appl. 345 (2008), 929-933. 